\title{
The influence of weave architecture on the mechanical properties of self-reinforced polypropylene
}

\author{
Y. Swolfs ${ }^{* 1}$, L. Crauwels ${ }^{1}$, L. Gorbatikh ${ }^{1}$, I. Verpoest ${ }^{1}$ \\ ${ }^{1}$ Department Metaalkunde en Toegepaste Materiaalkunde, KU Leuven, Kasteelpark \\ Arenberg 44 bus 2450, Belgium \\ *Corresponding author: Y. Swolfs (yentl.swolfs@mtm.kuleuven.be), Tel.: 00321632 \\ 1231
}

\section{Abstract}

The weave architecture is vital for hot compaction and the mechanical properties of self-reinforced polypropylene. Low compaction quality resulted in early damage initiation and reduced tensile strength. Interleaved films and decreased crimp in the weave architecture increased the compaction quality. The best compaction quality and tensile properties were obtained by standard fed weaves with interleaved films. The penetration impact resistance and peel strength was independent of the weave architecture. Interleaved films increased the peel strength drastically, but the impact resistance only slightly decreased. These conclusions help to select the correct weave architecture and facilitate the hot compaction process.

Keywords: A. fabrics/textiles; A. Thermoplastic resin; B. Mechanical properties; B. Impact behaviour

\section{Introduction}

Polymers are extensively used to replace materials such as metals and wood. Their low density, good processability and attractive price are key properties that contribute to their success. Fibrous reinforcements can be added to use polymers in structural applications. To increase the applicability in structural applications, fibrous reinforcements can be added. The resulting fibre-reinforced polymer composites possess excellent stiffness and strength and are now widely used in aerospace, transportation, sports and wind energy industries.

Creating a strong interface between fibres and polymers remains problematic, even though it remains a vital parameter for properties such as transverse strength and fatigue resistance. A solution for the limited mechanical properties of polymers and the low interfacial strength of composites was found in self-reinforced composites (SRC), also known as one polymer, all polymer or single polymer composites. Capiati and Porter [1] invented these composites in 1975, by exploiting the difference in melting point between highly oriented polyethylene (PE) filaments and unoriented PE with a lower molecular weight. Because matrix and fibre consist of the same polymer, the interface is well bonded. In contrast to isotropic PE, the highly oriented PE results in improved mechanical properties. 
Apart from the film stacking procedure of Capiati and Porter [1], researchers developed several other SRC processes [2-7]. An overview of these production process can be found in $[8,9]$. The two most important processes are hot compaction and co-extrusion. The former exploits the difference in melting temperature between the inner core and outer sheath of oriented polymer fibres $[3,10]$. Since the matrix is created by partially melting the fibre, molecular continuity between fibre and matrix is achieved. This continuity is expected to result in an even stronger interface than that of Capiati and Porter [1]. This interface is vital for impact performance, which is a key advantage of self-reinforced composites. The main disadvantage of hot compaction is the narrow processing window, which is limited to $2-4{ }^{\circ} \mathrm{C}$ [11-13]. This window can be widened by adding interleaved films $[14,15]$ or by switching to co-extrusion. Alcock and coworkers $[4,16,17]$ developed the latter technique to obtain a wide processing window for SRCs. The tape used in this process consists of an oriented homopolymer core and a thin copolymer skin. The difference in melting temperature between the homopolymer and copolymer is exploited to create the matrix and ensure proper bonding. This approach indeed results in a wider processing window of about $30^{\circ} \mathrm{C}$ [4]. The processing temperature is typically $10-20^{\circ} \mathrm{C}$ lower than that for hot compaction, resulting in a lower loss of molecular orientation and better tensile properties.

The impact resistance of SRCs is widely studied as it is a key advantage of SRCs [16, 18-22]. SRCs even have an increasing impact resistance with decreasing testing temperatures [16], which is remarkable material behaviour. The link between impact resistance and interlayer bonding strength is not established yet. Instead, the interlayer bonding strength is commonly assessed by peel tests [14, 15, 23, 24]. Literature describes three parameters that affect the peel strength. Firstly, Hine et al. [25] proved that the peel strength increases with compaction temperature. Since higher compaction temperature also decreases the mechanical properties by relaxation of the oriented tapes, a narrow optimal temperature window can be found. Secondly, the peel strength can be improved by adding interleaved films $[14,15]$. This helps widening the temperature window for hot compaction. Finally, Jordan et al. [23] proved that the interlayer bonding in tape weaves is much stronger than in multifilament weaves. Moreover, the authors mention that the weave architecture can affect the peel strength. In their work, the compaction temperature was different for all the tested configurations. Since compaction temperature is crucial for peel strength [25], it is not straightforward to derive the link between peel strength and weave architecture from the results of Jordan et al. [23].

The current paper investigates the link between the weave architecture and the mechanical properties of hot compacted self-reinforced polypropylene. Since the volume of voids depends on the weave architecture, each architecture will require a different amount of matrix to fill all the voids. To allow for a direct comparison, all tests will be performed on sheets that are hot compacted at the same temperature. The use of interleaved films to create additional matrix will be assessed, as this can help to fill the voids. By increasing the understanding of the various parameters affecting the hot compaction process, this paper aims to improve the compaction quality of SRCs in general. 


\section{Materials and methods}

\subsection{Materials}

Two types of drawn PP tapes were used, which were subjected to the same drawing conditions, but have different dimensions. The linear density of the tapes is 110 tex and 190 tex respectively. Both tapes were measured to have a stiffness of $6.9 \pm 1.2 \mathrm{GPa}$ and a strength of $589 \pm 24 \mathrm{MPa}$ [26].

These tapes were used to produce weaves at Propex Fabrics $\mathrm{GmbH}$ in plain (see figure 1a) and twill $2 / 2$ patterns (see figure $1 \mathrm{~b}$ ). A crucial aspect of tape weaving is the feeding: standard fed (SF) or overfed (OF). This is illustrated in figure 2. Since tapes have a high width/thickness ratio, they have the tendency to fold along their length during weaving. This can be exploited in weaving looms to obtain a higher production rate and increased areal density. In the warp direction, overfeeding can be achieved by having more tapes than would normally fit in. In the weft direction, the overfeeding is controlled by the battening after the weft insertion. If the weave is battened too hard, then the weft yarns will fold and become overfed (see figure 2). The overfeeding can be calculated as the ratio of the number of tapes per metre to 1000 divided by the width in millimetre:

$$
\text { overfeeding }=\frac{\# \text { tapes } / \mathrm{m}}{1000 / \text { width }(\mathrm{mm})} \cdot 100 \%
$$

The overfeeding can be different in both directions, but in the weaves used here, it was the same. The twill OF and plain OF weave both have an overfeeding of $50 \%$ in the warp and weft direction. Since folding increases the apparent tape thickness, the overfeeding also results in an increased crimp, which is a measure for the out-of-plane orientation of the tapes. The lower crimp of twill weaves can be seen from the higher amount of straight sections in figure 1b compared to figure 1a. To assess the influence of the overfeeding, a twill weave with standard feed (SF) was also woven. To reduce the probability of folded tapes and to maintain the same areal density and layer thickness, the standard fed weave uses the larger tape B. The areal density of the twill SF weave is $125 \mathrm{~g} / \mathrm{m}^{2}$, which is close to the $130 \mathrm{~g} / \mathrm{m}^{2}$ of the twill OF and $140 \mathrm{~g} / \mathrm{m}^{2}$ of the plain OF weave. Other weave configurations were not available.

Propex GmbH also provided $20 \mu \mathrm{m}$ thick films of the same PP grade, with a melting point of $163^{\circ} \mathrm{C}$. 


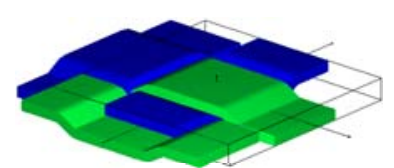

(a)

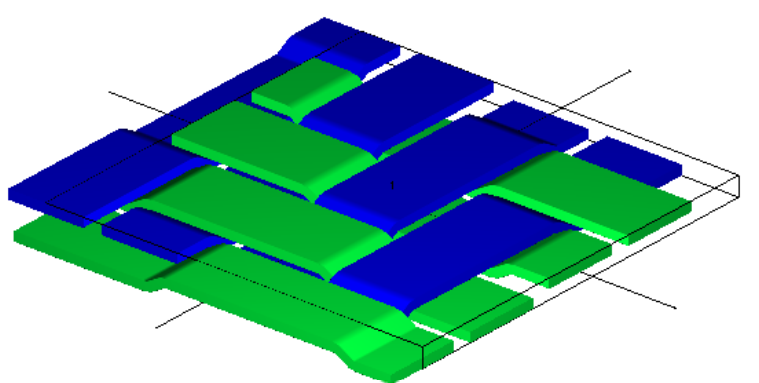

(b)

Figure 1: Schematic illustration of weave patterns without overfeeding: (a) plain, and (b) twill 2/2

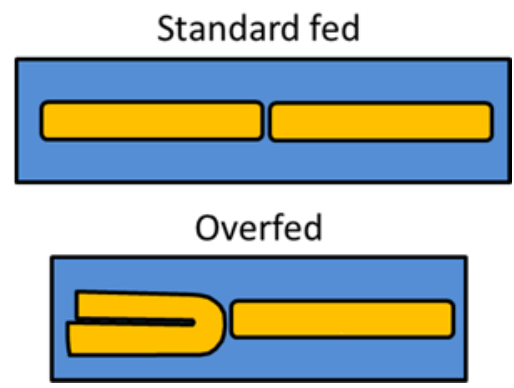

Figure 2: Schematic illustration of the difference between standard fed and overfed weaves. The overfed weaves contain tapes, which are folded along their length.

\subsection{Hot compaction}

For the tensile and impact samples, twelve woven layers of 320 x $320 \mathrm{~mm}$ were stacked onto each other and placed in between two $1 \mathrm{~mm}$ thick aluminium cover plates. The hot press was preheated at $188^{\circ} \mathrm{C}$ for 15 minutes to ensure a homogeneous temperature distribution over the press platens. The assembly was then inserted into the hot press at $188^{\circ} \mathrm{C}$. After a dwell time of 5 minutes, the press was cooled to $40^{\circ}$ in 5 minutes. The hot press applied a pressure of 40 bar during the entire cycle. All three weave architectures were processed according to this procedure. A fourth configuration was obtained by adding a single interleaved film between each layer of twill OF weave. This configuration is labelled "twill OF-F". These four configurations and their thicknesses are listed in table 1.

Table 1: Overview of the tensile and impact samples for the four configurations.

\begin{tabular}{cccccc}
\hline Label & Tape & Weave pattern & Overfeeding & Interleaved films & Thickness (mm) \\
\hline Twill OF & A & Twill 2/2 & Yes & No & $1.75 \pm 0.3$ \\
Plain OF & A & Plain & Yes & No & $1.89 \pm 0.2$ \\
Twill SF & B & Twill 2/2 & No & No & $1.64 \pm 0.1$ \\
Twill OF-F & A & Twill 2/2 & Yes & Yes & $1.96 \pm 0.3$ \\
\hline
\end{tabular}


A similar procedure was used to prepare peel strength samples. A $12 \mu \mathrm{m}$ polyimide release film was put on top of two woven layers, over the entire length of the weaves and over a depth of $90 \mathrm{~mm}$. Two woven layers were added on top of this stack and hot compacted under the same processing conditions.

\subsection{Differential scanning calorimetry}

Differential scanning calorimetry (DSC) samples were cut from the middle of the plates for tensile and impact tests. The samples, with a nominal weight of $5 \mathrm{mg}$, were tested in TA instruments Q2000. A 50/50 helium/nitrogen flow of $50 \mathrm{ml} / \mathrm{min}$ was used. At least four samples were tested for each configuration. The melting temperature was determined at the maximum of the heat capacity versus temperature.

\subsection{C-scan}

The compaction quality was evaluated by ultrasonic C-scans on the 320 x $320 \mathrm{~mm}$ plates for tensile and impact tests. The plates were immersed in demineralised water. Due to the thickness of the plates, the reflections from the glass plate beneath the samples were used. The scans were performed with an Olympus Panametrics V309SU transducer at a $5 \mathrm{MHz}$ frequency and $13 \mathrm{~mm}$ nominal diameter. The step size was $2 \mathrm{~mm}$ at a scan rate of $0.2 \mathrm{~mm} / \mathrm{s}$. Histograms were obtained from the measured greyscale values over a $200 \times 200 \mathrm{~mm}$ square in the middle of the scanned plates. Voids and unimpregnated zones shift the histogram to lower values and broaden the histogram.

\subsection{Tensile tests}

Tensile tests were performed according to ASTM D3039 on 250 x $25 \mathrm{~mm}$ samples with a gauge length of $150 \mathrm{~mm}$. The average thickness of these samples is summarised in table 1. A total of 9 samples were tested for each configuration. All tests were conducted on an Instron 4505 tensile machine, with a $100 \mathrm{kN}$ load cell and hydraulic clamps. The load cell was electronically scaled to $10 \mathrm{kN}$, to reach an accuracy of $5 \mathrm{~N}$. Sandpaper was used as end-tabs to avoid slippage in the clamps. The strain rate was $5 \% / \mathrm{min}$. The average surface strain was measured by strain mapping of the speckled surface.

The tensile modulus was calculated as the slope of the stress-strain diagram between 0,1 and $0,3 \%$ strain. The strength was calculated as the highest stress and the failure strain as the strain at which this occurs. The area underneath the stress-strain diagram was calculated by integrating the stress-strain diagram, resulting in a measure for the energy absorption per volume of material loaded.

\subsection{Falling weight impact tests}

Falling weight impact tests were performed according to ISO 6603-2. A hemispherical striker with $20 \mathrm{~mm}$ diameter was used in combination with a $40 \mathrm{~mm}$ inner clamping diameter. The $26.17 \mathrm{~kg}$ striker was dropped from $1 \mathrm{~m}$ height onto 100 x $100 \mathrm{~mm}$ specimens, which was sufficient to penetrate all samples. At least 10 specimens were tested for each configuration. The penetration impact energy was calculated as the area 
underneath the force-displacement diagram until the load has dropped below half of the peak load.

\subsection{Peel strength tests}

To evaluate the peel strength, T-peel tests were performed according to ASTM D1876. The 320 x $320 \mathrm{~mm}$ plate was cut into 20 x $300 \mathrm{~mm}$ samples by a sharp knife. The samples were cut in such a way that the length of the insert film, which is equivalent to the unbonded length, was always $76 \mathrm{~mm}$. The two unbonded ends were clamped by rubber clamps in the tensile machine Instron 5943 with a load cell of $1 \mathrm{kN}$ and pulled apart at a rate of $254 \mathrm{~mm} / \mathrm{min}$. The peel strength was defined as the peel load per $\mathrm{mm}$ width of the sample, in which the load was calculated as the average load over the first $127 \mathrm{~mm}$ displacement after the initial load peak. Ten samples were tested for each configuration.

\section{Results}

The quality of hot compacted SRCs is highly sensitive to the processing conditions. At the compaction temperature, the outer sheath of the tapes melts and this molten material recrystallises upon cooling to form the matrix. Due to the narrow temperature window for hot compaction, the compaction temperature has to be well chosen. The compaction temperature should be chosen in such a way that sufficient molten matrix is created to fill all empty space. As the volume of empty space depends on the weave architecture (see figure 1), each weave will require a different amount of matrix. The influence of the weave architecture on the mechanical properties will be investigated. This will be done under the same processing conditions for all configurations. The properties of interest are the tensile behaviour, the impact resistance and the peel strength. Before proceeding to the mechanical properties, the production quality will be assessed by DSC and C-scan.

\subsection{Production quality}

To ensure that all samples have experienced the same temperature and pressure cycle, all configurations have been verified by DSC. The melting peaks for the four different configurations as well as the PP film are presented in the DSC thermograms in figure 3. The melting peaks of the four configurations are similar, except for the configuration with interleaved films. No significant differences are found in the melting temperatures, see table 2. Nevertheless, two differences are observed for the configuration with interleaved films, which has a lower melting peak and contains a shoulder at about $160^{\circ} \mathrm{C}$. The shoulder is due to the additional matrix created by the interleaved films, as can be seen in figure 3. This matrix has a lower crystallinity and less perfect crystals than the tapes, which results in a slightly lower melting enthalpy for twill OF-F. The latter can be observed in table 2 . 


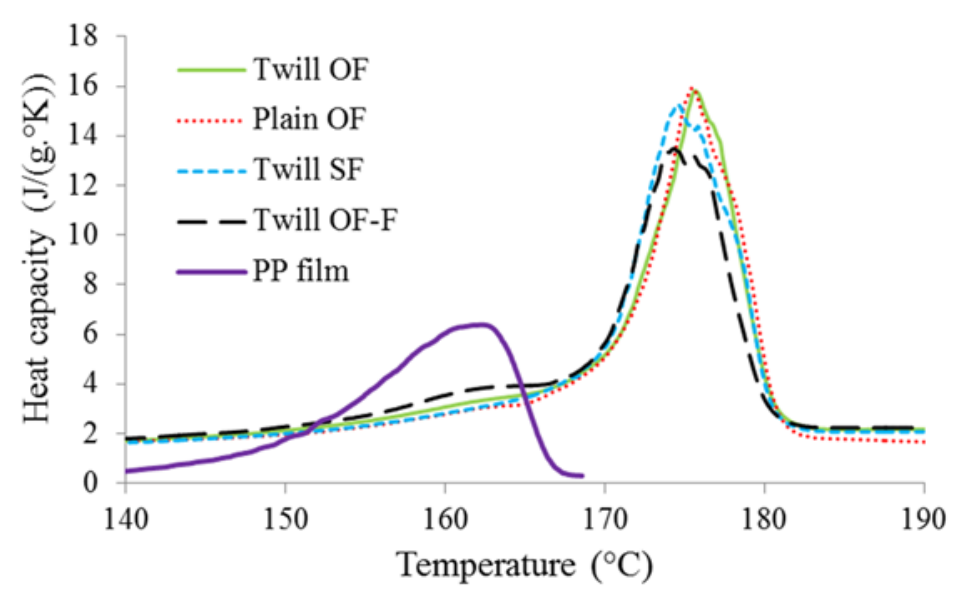

Figure 3: DSC thermograms for the different weave architectures. The thermogram for the PP film was added for a better comparison.

Table 2: Melting temperature and enthalpy for the four configurations.

\begin{tabular}{ccc}
\hline Label & Melting temperature $\left({ }^{\circ} \mathrm{C}\right)$ & Melting enthalpy $(\mathrm{J} / \mathrm{g})$ \\
\hline Twill OF & $176.2 \pm 1.2$ & $116.2 \pm 3.7$ \\
Plain OF & $177.5 \pm 1.7$ & $119.0 \pm 4.3$ \\
Twill SF & $175.8 \pm 0.5$ & $115.0 \pm 3.4$ \\
Twill OF-F & $175.2 \pm 1.8$ & $110.7 \pm 1.8$ \\
\hline
\end{tabular}

The DSC results prove that all configurations were subjected to the same thermal processing and that the results are reproducible. This means that any difference in mechanical properties cannot be directly attributed to a difference in thermal cycle.

A second quality verification tool is C-scan, a technique which is able to assess the compaction quality. The C-scan histograms in figure 4 illustrate the differences in compaction quality. A narrow peak at high intensities is found for the standard fed twill weave. Due to the lack of folded tapes, this weave has a flat surface and does not require much matrix to fill up all the voids. For this weave, the hot compaction process created sufficient matrix, resulting in a homogeneous quality. 


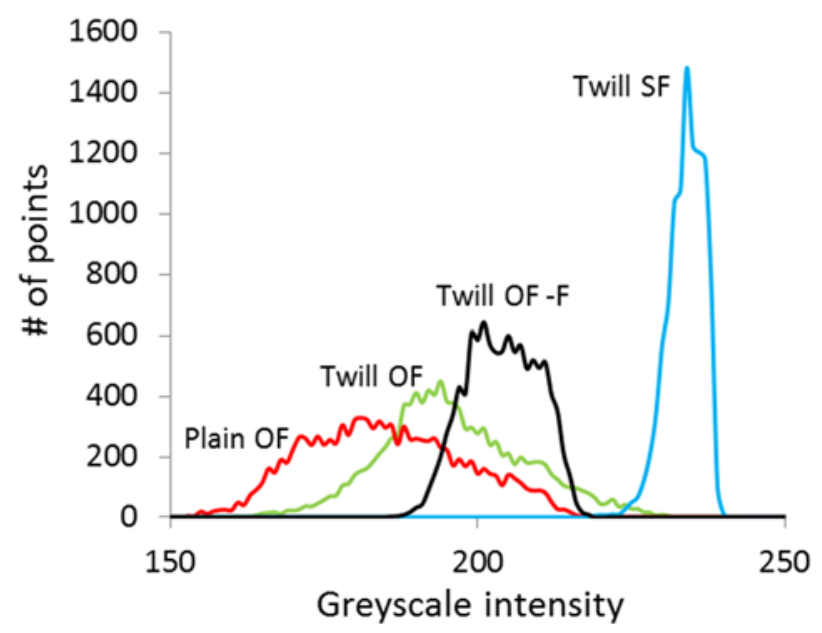

Figure 4: C-scan histogram to assess the impregnation quality of the different weave architectures.

The worst compaction quality is found in the plain OF weave. This weave has the highest crimp and requires more matrix to fill up all the voids caused by the overfeeding. The compaction temperature is not high enough to fill all the voids, which results in broader histogram at lower greyscale values (see figure 4). The reduced amount of crimp in the overfed twill weaves results in a higher compaction quality. By adding interleaved films, the compaction quality can be further increased, as more matrix is created and less voids remain. The additional matrix is still not sufficient to achieve compaction quality as high as in the twill SF. It seems that some of the voids created by the overfeeding can only be filled up if the temperature is further increased. This is undesired, as it will also increase molecular relaxation and decrease the mechanical properties.

In conclusion, these results illustrate that standard feeding and interleaved films facilitate achieving sufficient compaction quality, even at lower hot compaction temperatures. The standard feeding results in a lower amount of voids that need to be filled, while the interleaved films provide additional matrix to fill the voids. This decreases the lower temperature limit for hot compaction, which is determined by the compaction quality. The upper temperature limit, which is determined by molecular relaxation and tape melting, can be assumed to be unaffected by the weave architecture or interleaved films. This means that, although not directly proven, a wider temperature window for hot compaction is achieved.

\subsection{Tensile behaviour}

Figure 5 presents a representative tensile diagram for each of the four configurations. The tensile moduli of the four configurations are similar, see figure 6a. The only significant difference is found for Twill OF-F compared to Plain OF. Interleaved films add more matrix, which reduces the volume fraction of the tapes. The 11 films should in theory increase the thickness by $220 \mu \mathrm{m}$, which is close to the $210 \mu \mathrm{m}$ difference between twill OF and twill OF-F (see table 1). If the stiffness of the film is assumed to be $1.7 \mathrm{GPa}$, then the linear rule-of-mixtures predicts a tensile modulus of $3.01 \mathrm{GPa}$. This prediction lies within the 95\% confidence interval of the tensile modulus of twill OF-F, which is $3.06 \pm 0.05 \mathrm{GPa}$. 


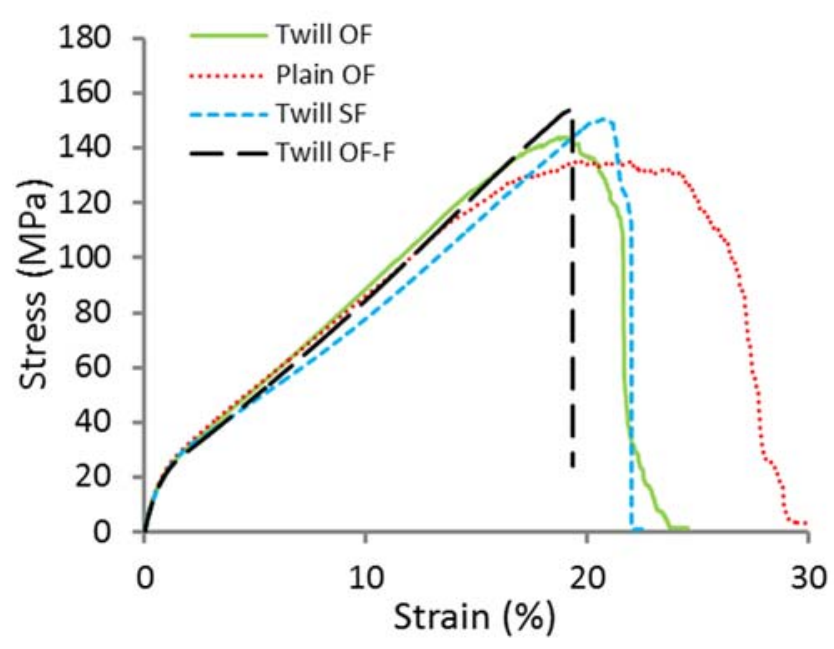

Figure 5: Tensile behaviour of SRPP with different weave architectures.

At a stress level of about $20 \mathrm{MPa}$, all configurations start yielding. This can also be observed in single tape tensile diagrams [3, 27], which is an indication that it is not caused by straightening of the tapes. The yielding is caused by the tie molecules which connect the highly oriented crystallites in the drawn tapes. At the yield point, these tie molecules start to flow, which explains the decrease in stiffness. The tie molecules gradually increase their orientation in the tensile direction, and hence the stress further increases. The stiffness reduction in this region is larger for the standard fed twill. It is not clear why this is the case.

At $15 \%$ strain or higher, deviations from linear behaviour start to appear, which coincides with the appearance of damage in the samples. The damage initiates by debonding of the tapes from the matrix and delaminations in between the layers. Tape fracture is only observed after the stress has reached its maximum. In the plain weave, damage initiates at about $15 \%$ strain and the strain interval over which damage is accumulated is more than $15 \%$. In the twill weaves without interleaved films, the damage initiation happens a few percent of strain later and over a smaller strain interval. In the samples with interleaved films, debonding or delamination was not observed. In samples without interleaved films, this can be visually observed by the transition from semi-transparent to white samples. This results in a linear stress-strain diagram after the yield point, as well as a higher strength, see figure 6b. The final failure happens suddenly, without any visible damage prior to failure. The twill SF configuration demonstrates some visible damage prior to failure, but not as much as twill OF.

Even though differences in strength are observed in figure 6b, the strain at which this strength is achieved is the same for all configurations, see figure 6c. Finally, figure 6d compares the absorbed energy per volume of loaded material. On one hand, the abrupt failure of the weaves with interleaved films results in the smallest energy absorption. On the other hand, the more gradual failure of the plain weave spreads out the damage over a larger strain interval and results in a larger energy absorption. 

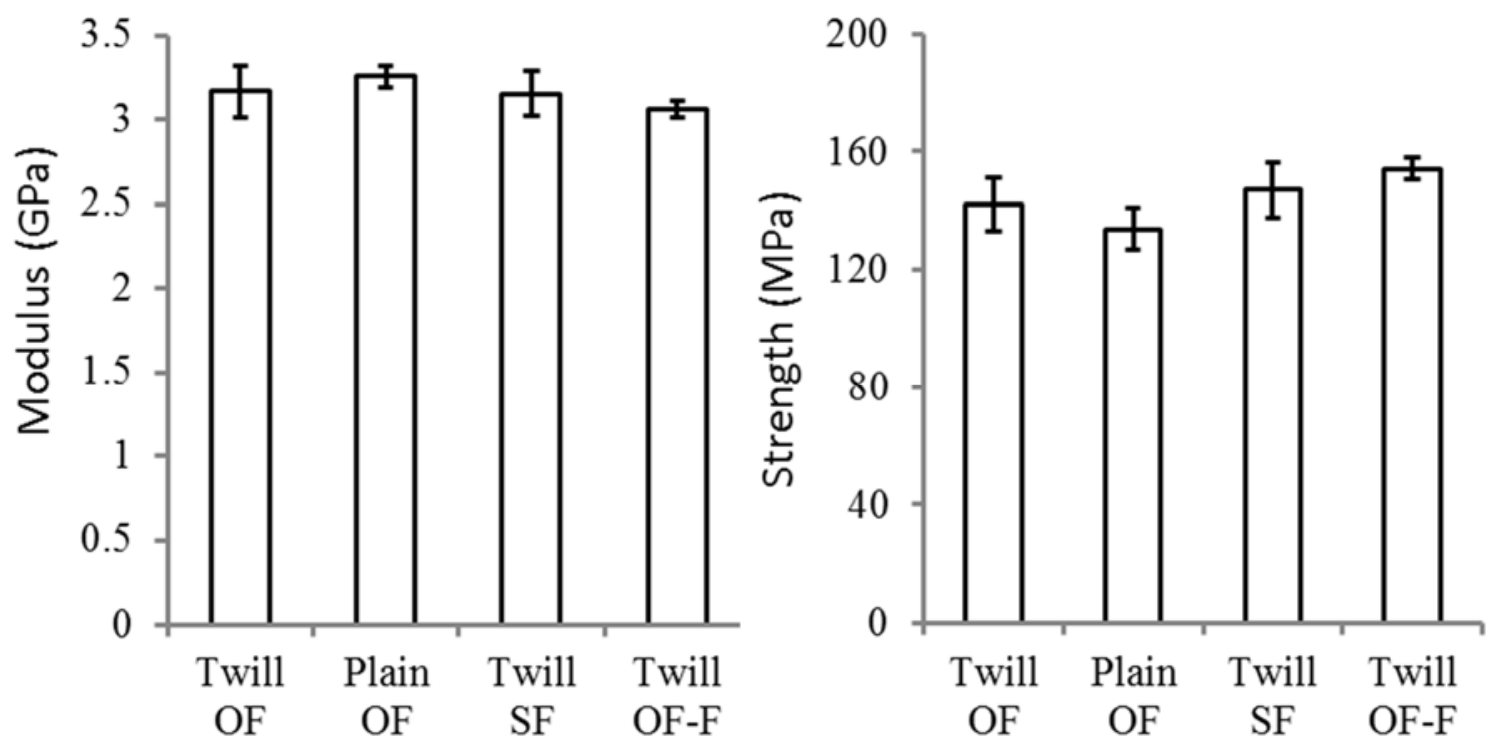

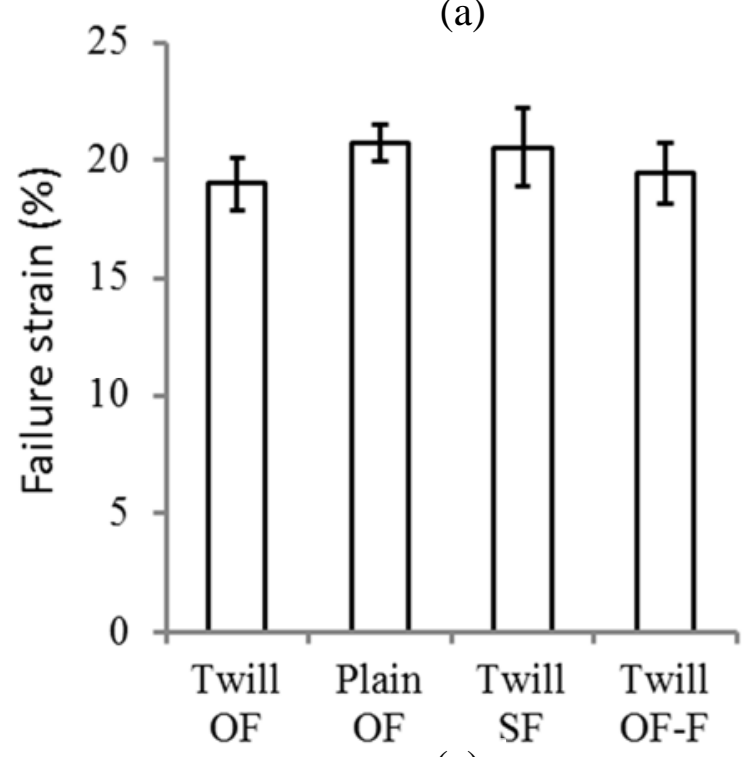

(c)

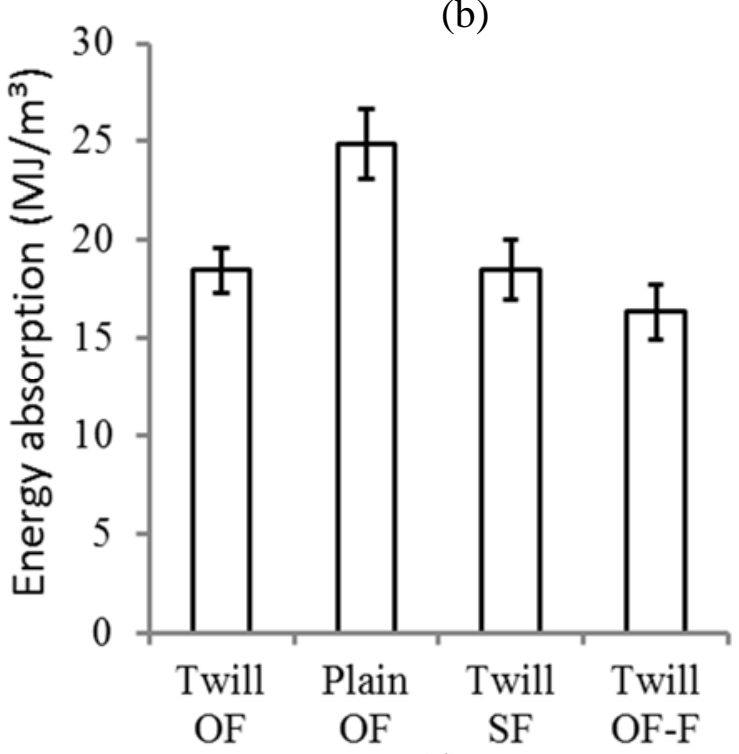

(d)

Figure 6: Tensile results of different weave architecture: (a) modulus, (b) strength, (c) failure strain, and (d) area underneath the stress-strain diagram.

\subsection{Impact behaviour}

On top of the good tensile properties of SRPP, the impact resistance is a vital parameter for many applications. Penetration impact testing has become a standard test procedure to evaluate the impact resistance of SRCs. Figure 7 summarises the impact resistance of the different configurations. No significant differences are found in the impact resistance per mm of sample thickness for the weaves without interleaved films. As stated by Aurrekoetxea et al. [18] and Alcock et al. [19], the penetration impact resistance of well compacted SRCs is dominated by tape fracture and not by delaminations or debonding. 


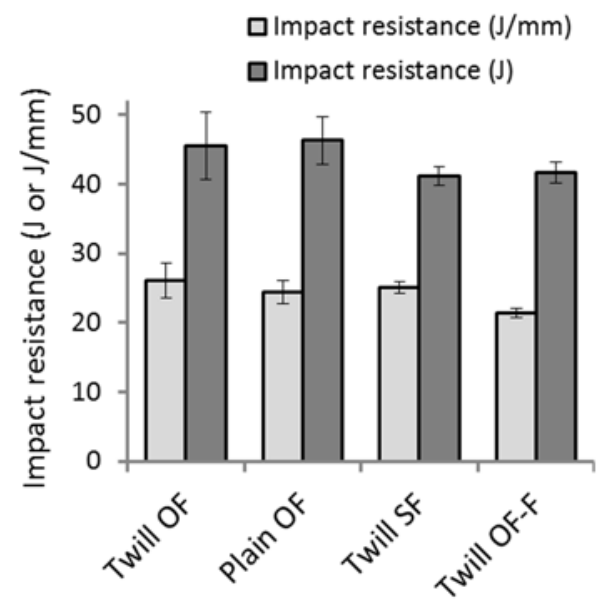

Figure 7: Impact resistance per mm of sample thickness and in absolute values.

Even though twill OF and twill OF-F contain the same amount of tapes that can absorb energy, the twill OF-F does have a lower impact resistance per mm thickness (see figure 7 ). The impact resistance per mm thickness is decreased $18 \%$ by the interleaved films. This decrease is caused by the two effects. Firstly, the additional films increase the sample thickness, see table 1 . The twill OF-F is $12 \%$ thicker than the twill OF configuration, while the isotropic PP film only absorbs a relatively small amount of energy compared to the oriented PP tapes. If the impact resistance is expressed in absolute terms, then the difference reduces to $8 \%$ (see figure 7 ). The p-value of this difference is $8.5 \%$ in a two-tailed t-test. Secondly, the interleaved films improve the compaction quality by adding more matrix. This increases the strength of the interlayer bonding and reduces the amount of debonding and delamination in impact tests, resulting in a lower energy absorption during penetration impact. The fact that the difference in absorbed energy is small, confirms that debonding and delaminations only play a small role in the energy absorption during penetration impact tests.

\subsection{Peel strength}

To further understand the tensile behaviour and impact resistance, peel strength tests were performed. This yields information on the force needed to delaminate two layers of the weave. The peel strength relates to mode I type of delamination, in which the force is perpendicular to the crack propagation direction. In impact tests, mode II is dominant, because the delaminations in impact tests are caused by shear stresses. Nevertheless, peel strength is a good measure of the influence of the resistance a material has against delamination. Figure 8 illustrates a representative peel load diagram for each configuration, while figure 9 presents the resulting peel strengths. 


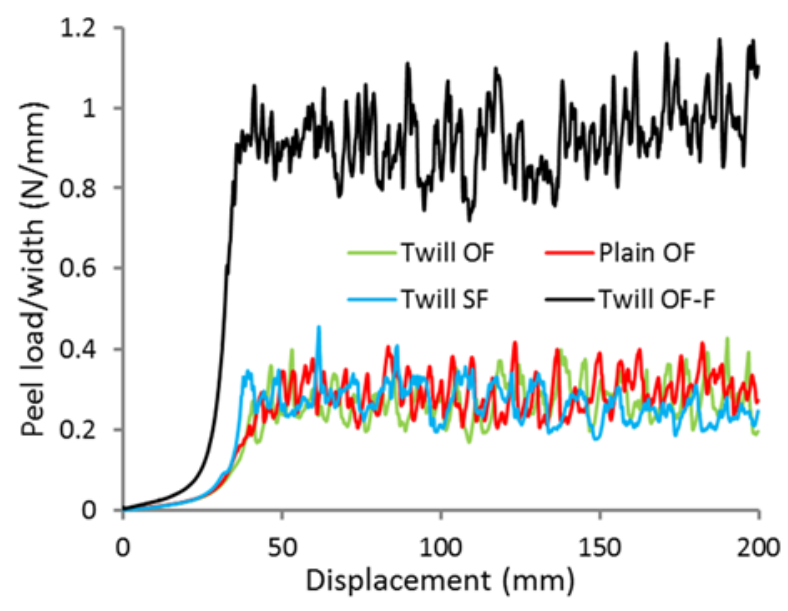

Figure 8: Example of a peel load diagram for each configuration, in which peel load is divided by sample width and plotted as a function of displacement.

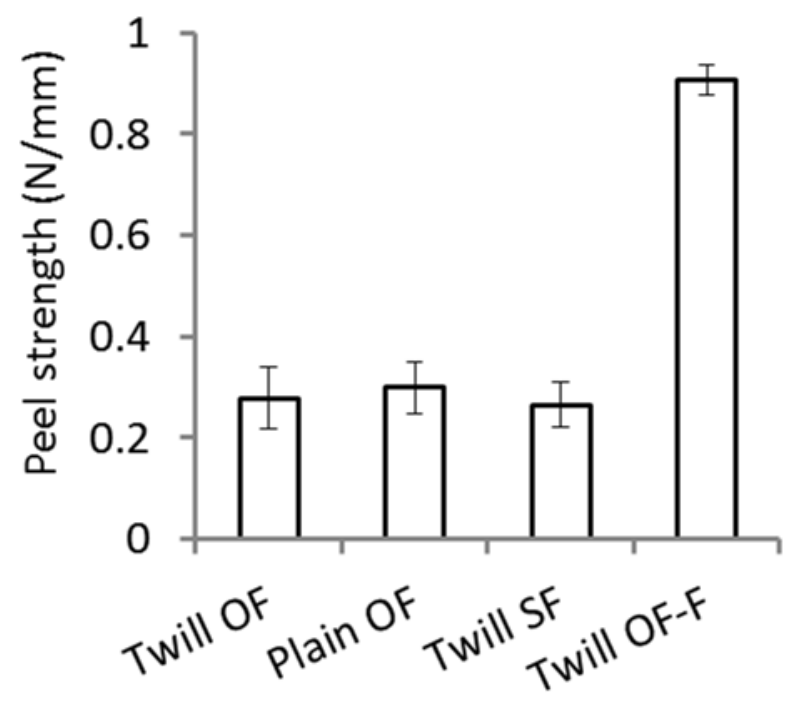

Figure 9: Peel strength for each configuration.

No significant differences in peel strength can be discerned for samples without interleaved films. On one hand, the twill SF configuration has better compaction quality, which should result in higher peel strength. On the other hand, the twill SF has a flat and smooth surface, which facilitates delamination and debonding compared to the rough surface of the overfed weaves. These two phenomena seem to balance each other out and result in similar peel strength. This is also confirmed by the stereomicroscopic images of the peel surfaces in figure 10. The white regions reflect the presence of damage created by the debonding and delamination. Most of the damage is concentrated on the transverse tapes, as they are the weakest in the peeling direction.

The configuration with interleaved films has significantly higher peel strength, and this is also visible in the peel surface. More white regions are observed in figure 10d and they are also present on the tapes in the peeling direction. This indicates good bonding between the layers and means that interleaved films increase the required energy to delaminate SRPP. 

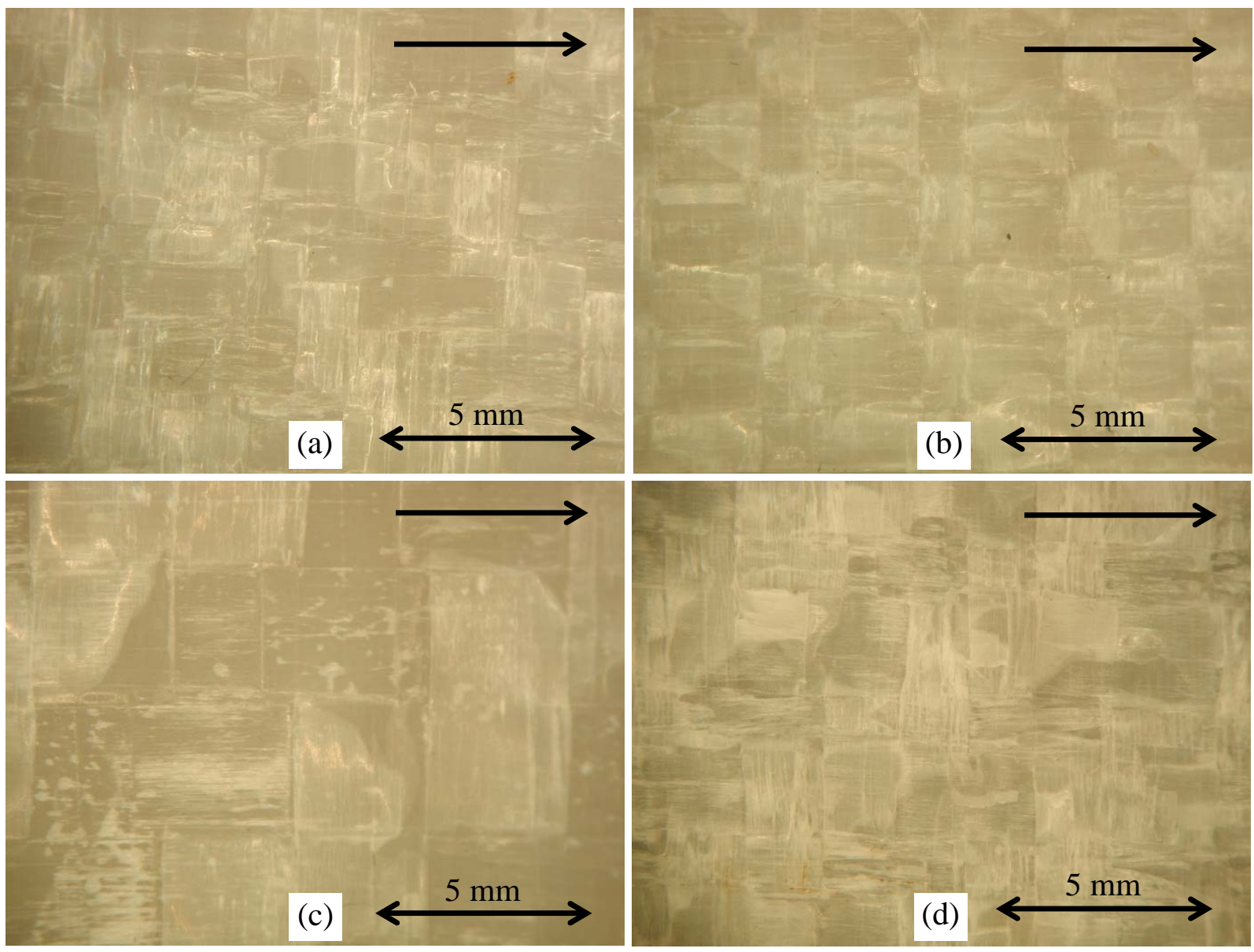

Figure 10: Stereomicroscopic images of the peel surfaces of (a) twill OF, (b) plain OF, (c) twill SF, and (d) twill OF-F. The arrow indicates the peeling direction.

\section{Discussion}

Even though C-scan results demonstrated significant compaction quality differences, the peel strengths and impact resistance are the same for all configurations without interleaved films. This means that both properties are less sensitive to voids and are dominated by other mechanisms. Peel strength in SRCs is dominated by debonding and delamination mechanisms, while impact resistance is dominated by tape fracture and fibrillation. In contrast, tensile behaviour depends on the compaction quality. Voids or areas with insufficient matrix lead to earlier damage initiation and lower strength. The strength of the oriented tapes can only be fully exploited if the compaction quality is high.

Only a small fraction of the energy absorbed during penetration is absorbed by delaminations and debonding. This means that the penetration impact of SRPP is dominated by other energy absorption mechanisms. The fracture of the tapes and the accompanying fibrillation are likely to be the dominating mechanism in hot compacted SRPP. This confirms the findings of Aurrekoetxea et al. [18] for hot compacted SRPP. Interestingly, Alcock et al. [19] reported that penetration impact of co-extruded SRPP is dominated by a combination of delamination and tape fracture. Delamination dominates 
at low compaction temperatures, while tape fracture dominates at higher compaction temperatures. Moreover, Barany et al. [21] reported the same parameters for SRPP produced by film stacking. These results suggest that hot compacted SRPP may be less prone to delaminations than other SRC processes.

Finally, to obtain a high compaction quality, the weave architecture should be taken into account. As described in literature [23, 27], tapes are preferred over fibres as less voids need to be filled up during hot compaction. These conclusions can be extended to two parameters of weave architectures: overfeeding and crimp. Overfeeding, which is used in industry to increase the production rate of weaving looms, also results in weaves with a rough surface. These weaves require a large amount of matrix to fill up the voids. Crimp has a similar influence, as it increases the waviness of the tapes and thereby increases the required amount of matrix. Therefore, a weave with a flatter surface can be hot compacted at lower temperatures, which means that molecular relaxation can be reduced and mechanical properties can be improved. The processing window can be further enlarged if interleaved films are added, but this has the disadvantage of slightly reducing the impact resistance.

\section{Conclusion}

The tensile behaviour and impact resistance of hot compacted self-reinforced polypropylene (SRPP) was evaluated for three different weave architectures: overfed twill, overfed plain and standard fed twill. The influence of interleaved films was also assessed for the overfed twill weave. The compaction quality increases with a decrease in the crimp. Lower crimp allows a lower compaction temperature and thereby widens the temperature window for hot compaction. This can aid in choosing suitable weaves for hot compaction.

- The tensile behaviour is affected by the compaction quality. Lower compaction quality results in earlier damage initiation, lower strength and more extensive damage. If interleaved films are used, the compaction quality is higher and final failure occurs more sudden and at higher stress.

- The penetration impact resistance slightly decreases by adding interleaved films, but remains unaffected by the weave architecture.

- The peel strength increases by adding interleaved films, but remains unaffected by the weave architecture.

The higher peel strength for the samples with interleaved films proves the higher energy required delaminations. Since this is not reflected in the impact resistance, delaminations play only a minor role in the energy absorption during penetration impact of hot compacted SRPP.

Future work should clarify how peel strength, impact resistance and compaction quality evolve with compaction temperature. This can further strengthen the conclusions regarding the width of the temperature window for hot compaction and how it can be enlarged. More research is also needed in the damage development of SRCs to further understand how they fail and which parameters affect this failure behaviour. 


\section{Acknowledgements}

The work leading to this publication has received funding from the European Union Seventh Framework Programme (FP7/2007-2013) under the topic NMP-2009-2.5-1, as part of the project HIVOCOMP (Grant Agreement No. 246389). The authors thank the Agency for Innovation by Science and Technology in Flanders (IWT) for the grant of Y. Swolfs and acknowledge the help of technicians Kris Van de Staey, Bart Pelgrims and Danny Winant. I. Verpoest holds the Toray Chair in Composite Materials at KU Leuven.

\section{References}

[1] Capiati NJ, Porter RS. The concept of one polymer composites modelled with high density polyethylene. Journal of Materials Science. 1975;10(10):1671-7.

[2] Barkoula NM, Peijs T, Schimanski T, Loos J. Processing of single polymer composites using the concept of constrained fibers. Polymer Composites. 2005;26(1):114-20.

[3] Ward IM, Hine PJ. The science and technology of hot compaction. Polymer. 2004;45(5):1413-27.

[4] Alcock B, Cabrera N, Barkoula N, Spoelstra A, Loos J, Peijs T. The mechanical properties of woven tape all-polypropylene composites. Composites Part A: Applied Science and Manufacturing. 2007;38(1):147-61.

[5] Izer A, Bárány T, Varga J. Development of woven fabric reinforced allpolypropylene composites with beta nucleated homo- and copolymer matrices. Composites Science and Technology. 2009;69(13):2185-92.

[6] Kmetty Á, Bárány T, Karger-Kocsis J. Injection moulded all-polypropylene composites composed of polypropylene fibre and polypropylene based thermoplastic elastomer. Composites Science and Technology. 2012;73(0):72-80.

[7] Kmetty A, Tabi T, Kovacs JG, Barany T. Development and characterisation of injection moulded, all-polypropylene composites. Express Polymer Letters. 2013;7(2):134-45.

[8] Kmetty Á, Bárány T, Karger-Kocsis J. Self-reinforced polymeric materials: A review. Progress in Polymer Science. 2010;35(10):1288-310.

[9] Fakirov S. Nano- and Microfibrillar Single-Polymer Composites: A Review. Macromolecular Materials and Engineering. 2013;298(1):9-32.

[10] Olley RH, Bassett DC, Hine PJ, Ward IM. Morphology of compacted polyethylene fibers. Journal of Materials Science. 1993;28(4):1107-12.

[11] Hine PJ, Ward IM, Teckoe J. The hot compaction of woven polypropylene tapes. Journal of Materials Science. 1998;33(11):2725-33.

[12] Teckoe J, Olley RH, Bassett DC, Hine PJ, Ward IM. The morphology of woven polypropylene tapes compacted at temperatures above and below optimum. Journal of Materials Science. 1999;34(9):2065-73.

[13] Hine PJ, Bonner M, Brew B, Ward IM. Hot compacted polypropylene sheet. Plastics Rubber and Composites Processing and Applications. 1998;27(4):167-71.

[14] Hine PJ, Unwin AP, Ward IM. The use of an interleaved film for optimising the properties of hot compacted polyethylene single polymer composites. Polymer. 2011;52(13):2891-8. 
[15] Hine P, Olley R, Ward I. The use of interleaved films for optimising the production and properties of hot compacted, self reinforced polymer composites. Composites Science and Technology. 2008;68(6):1413-21.

[16] Alcock B, Cabrera NO, Barkoula NM, Wang Z, Peijs T. The effect of temperature and strain rate on the impact performance of recyclable all-polypropylene composites. Composites Part B-Engineering. 2008;39(3):537-47.

[17] Alcock B, Cabrera NO, Barkoula NM, Loos J, Peijs T. Interfacial properties of highly oriented coextruded polypropylene tapes for the creation of recyclable allpolypropylene composites. Journal of Applied Polymer Science. 2007;104(1):118-29.

[18] Aurrekoetxea J, Sarrionandia M, Mateos M, Aretxabaleta L. Repeated low energy impact behaviour of self-reinforced polypropylene composites. Polymer Testing. 2011;30(2):216-21.

[19] Alcock B, Cabrera NO, Barkoula NM, Peijs T. Low velocity impact performance of recyclable all-polypropylene composites. Composites Science and Technology. 2006;66(11-12):1724-37.

[20] Fabich B, Taketa I, Gorbatikh L, Lomov SV, Janetzko S, Gries T, et al. Toughness improvement in hybrid composites made of carbon fibre reinforced polypropylene and self-reinforced polypropylene. Lancaster: Destech Publications, Inc; 2010.

[21] Bárány T, Izer A, Karger-Kocsis J. Impact resistance of all-polypropylene composites composed of alpha and beta modifications. Polymer Testing. 2009;28(2):176-82.

[22] Hine PJ, Ward IM. Hot compaction of woven poly(ethylene terephthalate) multifilaments. Journal of Applied Polymer Science. 2004;91(4):2223-33.

[23] Jordan ND, Bassett DC, Olley RH, Hine PJ, Ward IM. The hot compaction behaviour of woven oriented polypropylene fibres and tapes. II. Morphology of cloths before and after compaction. Polymer. 2003;44(4):1133-43.

[24] Foster RJ, Bonner MJ, Ward IM. The use of nano and micron-sized particles to enhance the interlayer adhesion in self-reinforced, single-polymer composites. Composites Science and Technology. 2011;71(4):461-5.

[25] Hine PJ, Ward IM, El Matty MIA, Olley RH, Bassett DC. The hot compaction of 2-dimensional woven melt spun high modulus polyethylene fibres. Journal of Materials Science. 2000;35(20):5091-9.

[26] Pinxten W. De invloed van het visco-elastisch gedrag van vertrekt polypropyleen op het krimpgedrag van polypropyleencomposieten [Master thesis]. Leuven: KU Leuven; 2005.

[27] Hine PJ, Ward IM, Jordan ND, Olley R, Bassett DC. The hot compaction behaviour of woven oriented polypropylene fibres and tapes. I. Mechanical properties. Polymer. 2003;44(4):1117-31. 